of referring the plane to lines or lineoids as coördinate elements, to determine the plane by reference to planes, as follows: if from the plane assemblage in question six planes $\pi_{1}, \cdots, \pi_{6}$ be chosen such that they furnish three pairs $\pi_{1} \pi_{6}, \pi_{2} \pi_{5}, \pi_{3} \pi_{4}$ of absolutely perpendicular planes, then we may take for homogeneous plane coördinates of a plane $\pi$ the six cosine products $\cos \theta_{a} \cos \omega_{\alpha}$, where $\theta_{\alpha}$ and $\omega_{a}$ are the angles of $\pi$ with the coördinate plane $\pi_{a}$.

The six coördinate planes intersect an arbitrary lineoid of 4-space in the six edges of a tetraedron, while the arbitrary plane $\pi$ cuts from the lineoid an equally arbitrary line. The angles $\theta$ and $\omega$ correspond to the distances and angles of the line with the tetraedral lines. The corresponding interpretation of the Plücker coördinates would accordingly be one in terms of these distances and angles.

We will close this note with the necessary and sufficient condition that two planes $\left({ }_{a}, \omega_{a}\right)$ and $\left(\theta_{a}{ }^{\prime}, \omega_{a}{ }^{\prime}\right)$ shall have a common line. It is

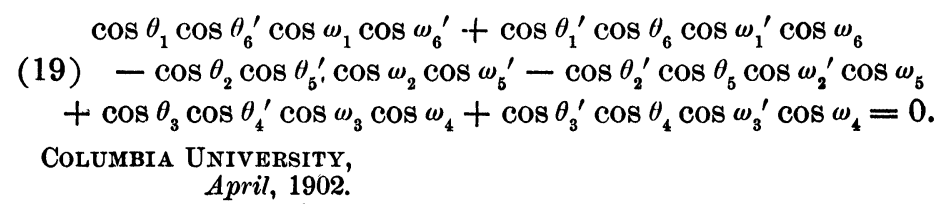

\title{
NOTE ON THE SUFFICIENT CONDITIONS FOR AN ANALYTIC FUNCTION.
}

BY MR. D. R. CURTISS.

(Read before the American Mathematical Society, April 26, 1902.)

Since the publication of Goursat's proof $*$ that a function of the complex variable $z$ possessing a derivative at each point of a two-dimensional region $T$ in which it is singlevalued must necessarily have a continuous derivative throughout that region, the question has arisen whether the sufficient conditions for an analytic function, stated in terms of the partial derivatives of the real and pure imaginary parts of the function, may not be reduced to simpler terms. These conditions are ordinarily given as follows:

$w=u(x, y)+i v(x, y)$ is an analytic function of the complex variable $z=x+i y$ at each point of a region $T$ of the $z$-plane if throughout $T$

\footnotetext{
* Trans. Am. Math. Soc., Vol. 1 (1900), No. 1, p. 14.
} 
1) $u$ and $v$ are single-valued, continuous functions of the independent real variables $x$ and $y$;

exist ;

$$
\frac{\partial u}{\partial x}, \frac{\partial u}{\partial y}, \quad \frac{\partial v}{\partial x}, \frac{\partial v}{\partial y}
$$

3) the Cauchy-Riemann differential equations

are satisfied ;

$$
\frac{\partial u}{\partial x}=\frac{\partial v}{\partial y}, \quad \frac{\partial u}{\partial y}=-\frac{\partial v}{\partial x}
$$

4) these four partial derivatives are continuous functions of the independent variables $x$ and $y$.

That condition 4) demands more than is necessary to prove the existence of a derivative of $w$ has been noted by Harnack, * and later by Stolz, $\dagger$ both writers showing that in its place may be substituted the requirement that $u$ and $v$ each possess a total differential. So far as I have been able to ascertain, no further substantial simplification of condition 4) has ever been published.

The result which I have obtained is that conditions 1), 2), $3)$, together with the continuity of ONE of the four partial derivatives of 2), are sufficient for the existence of a derivative $\frac{d w}{d z}$ and hence, by Goursat's theorem, are sufficient conditions that $w$ be an analytic function of $z$ throughout the region $T$.

Let $z_{0}=x_{0}+i y_{0}$ be any point in $T$; and let $w=u(x, y)$ $+i v(x, y)$ be a function whose real and imaginary parts, $u$ and $v$, satisfy conditions 1), 2), and 3). Further, assume $\frac{\partial u}{\partial x}$ continuous in both $x$ and $y$ at each point of $T$. Then at the point $\left(x_{0}, y_{0}\right)$ we have

$$
\begin{gathered}
\Delta u=u\left(x_{0}+\Delta x, y_{0}+\Delta y\right)-u\left(x_{0}, y_{0}+\Delta y\right)+u\left(x_{0}, y_{0}+\Delta y\right) \\
-u\left(x_{0}, y_{0}\right) \\
=\frac{\partial}{\partial x} u\left(x_{0}+\theta_{1} \Delta x, y_{0}+\Delta y\right) \Delta x+\frac{u\left(x_{0}, y_{0}+\Delta y\right)-u\left(x_{0}, y_{0}\right)}{\Delta y} \Delta y \\
\left(0<\theta_{1}<1\right) .
\end{gathered}
$$

On the hypothesis that $\frac{\partial u}{\partial x}$ is continuous and $\frac{\partial u}{\partial y}$ exists, we have then

* Differential- und Integral-Rechnung, p. 152.

† Differential- und Integral-Rechnung, vol. II, p. 82. 


$$
\Delta u=\left(\frac{\partial u}{\partial x}+\zeta_{1}\right) \Delta x+\left.\left(\frac{\partial u}{\partial y}+\zeta_{2}\right) \Delta y\right|_{x_{0}, y_{0}},
$$

where $\zeta_{1}$ and $\zeta_{2}$ approach the limit zero when $\Delta x$ and $\Delta y$, independently of each other, approach the limit zero.

From equations 3 ), $\frac{\partial v}{\partial y}$ must be continuous, hence we may write

$\Delta v=v\left(x_{0}+\Delta x, y_{0}+\Delta y\right)-v\left(x_{0}+\Delta x, y_{0}\right)$

and by reasoning as before,

$$
+v\left(x_{0}+\Delta x, y_{0}\right)-v\left(x_{0}, y_{0}\right)
$$

$$
\Delta v=\left(\frac{\partial v}{\partial y}+\zeta_{8}\right) \Delta y+\left.\left(\frac{\partial v}{\partial x}+\zeta_{4}\right) \Delta x\right|_{x_{0}, y_{0}},
$$

where $\zeta_{3}$ and $\zeta_{4}$ approach the limit zero when $\Delta x$ and $\Delta y$, independently of each other, approach the limit zero.

Using these expressions for $J u$ and $\Delta v$, we have the following form for the difference-quotient $\frac{\Delta w}{\Delta z}$;

$$
\left.\frac{\Delta w}{\Delta z}\right|_{x_{0}}=\frac{\Delta u+i \Delta v}{\Delta x+i \Delta y}=\frac{\left(\frac{\partial u}{\partial x}+i \frac{\partial v}{\partial x}\right) \Delta x+\left(\frac{\partial u}{\partial y}+i \frac{\partial v}{\partial y}\right) \Delta y}{\Delta x+i \Delta y}+\eta
$$

where the derivatives are formed for the point $\left(x_{0}, y_{0}\right)$, and $\eta$ approaches the limit zero with $\Delta x$ and $\Delta y$.

The first term on the right-hand side can, with the aid of condition (3), be thrown into the form $\frac{\partial u}{\partial x}+i \frac{\partial v}{\partial x}$. Hence $\frac{\Delta w}{\Delta z}$ approaches a limit when $\Delta z$ approaches zero, and

$$
\lim _{\Delta z=0} \frac{\Delta w}{\Delta z}=\left.\frac{d w}{d z}\right|_{z_{0}}=\frac{\partial u}{\partial x}+\left.i \frac{\partial v}{\partial x}\right|_{x_{0}, y_{0}} .
$$

We have thus proved the existence of a derivative, assuming the continuity of only one of the partial derivatives of (2). From the nature of the proof it is seen that this partial derivative might have been any one of the four.

The question still remains unanswered as to whether we may not dispense with any assumption concerning the continuity of the partial derivatives of $u$ and $v$.

Harvard University, Cambridge, Mass., February, 1902. 